\title{
Physical activity in premenopausal women with asymptomatic peripheral arterial disease Anna Vittoria Mattioli ${ }^{a}$, Francesca Coppi ${ }^{b}$, Mario Migaldi ${ }^{a}$ and Alberto Farinetti ${ }^{a}$
}

J Cardiovasc Med 2018, 19:677-680

Keywords: ankle-brachial index, antioxidants, diet, physical activity, women

${ }^{a}$ Department of Surgical, Medical and Dental Department of Morphological Sciences related to Transplant, Oncology and Regenerative Medicine, University of Modena and Reggio Emilia and ${ }^{\mathrm{b}}$ Cardiology Division, Azienda Ospedaliera

Universitaria, Modena, Italy

Correspondence to Anna Vittoria Mattioli, Professor, Department of Surgical, Medical and Dental Department of Morphological Sciences related to Transplant, Oncology and Regenerative Medicine, University of Modena and Reggio Emilia, Via del pozzo, 7141100 Modena, Italy

Tel: +3959 4224043; fax: +3959 4224323;

e-mail: annavittoria.mattioli@unimore.it

Received 24 July 2018 Accepted 16 August 2018

\section{Letter to the editor}

Lifestyle, including nutrition and physical activity, plays an important role in the development of cardiovascular disease (CVD) and may contribute to the geographic and sex variability in CVD morbidity and mortality. ${ }^{1,2}$ Regular exercise in combination with specific dietary patterns (i.e. Mediterranean pattern) may have a beneficial effect on the cardiovascular system through the modulation of the oxidation process. ${ }^{3,4}$

The ATTICA study had clearly shown that highly active people who exercise several days per week enjoyed greater protection from the oxidative process because of a higher total antioxidant capacity (TAC) level. ${ }^{5}$

We also reported that women with high adherence to a Mediterranean diet are less likely to develop preclinical atherosclerosis compared with women with a low adherence. ${ }^{4}$ The present study aims to evaluate the role of physical activity in women with high adherence to a Mediterranean diet and its correlation with asymptomatic atherosclerosis.

\section{Methods}

A retrospective analysis on a group of 650 women (age range $45-54$ years) was performed. Patients were refered to our clinic from general practitioners for screening and prevention of CVD. We selected women only if they were free of symptoms of peripheral arterial disease (PAD), had $\mathrm{ABI}$ evaluation and a complete nutritional assessment. We excluded participants with a previous history of cardiovascular disease (ischemic heart disease heart failure and stroke), those who did not complete a questionnaire, and those who did not undergo ABI evaluation and who did not sign the consent (Figure 1 supplemental material, http://links.lww.com/JCM/A141). The group of women who completed the physical activity questionnaire included 425 women (mean age $48 \pm 9$ years).

Study was approved by the Local Ethical Review Board and participants signed an informed consent.

\section{Data collection}

Nutritional status was assessed by measuring weight, body mass index (BMI), waist circumference and waist-to-hip ratio. ${ }^{6}$

The prevalence of nutritional parameters was assessed by a self-administered food frequency questionnaire $(\mathrm{FFQ})$ with 116 items and completed by an interviewer-administered 7-day diet recall questionnaire on the day of first visit. ${ }^{6}$

The food list in the FFQ was Italianized, and foods commonly eaten in the Emilia Romagna region of Italy were added. For each food class, color photographs of three different portions were displayed. Portion sizes were chosen according to many years of experience in dietary surveys in various parts of Italy. ${ }^{6}$

Food frequency was evaluated using three categories: daily, weekly and monthly and from one to six times (i.e. once a day, three times a week) and was integrated with specific questions on changes in nutrition habits and lifestyle within the last year. The Mediterranean score was calculated according to Panagiotakos. ${ }^{6}$ A dietician, using a database system, computed food and nutrient intakes from FFQ and dietary recall. The nutrient database was compiled from food composition tables.

\section{Physical activity}

We utilized the International Physical Activity Questionnaire (IPAQ) developed by the World Health Organization (WHO) and validated for the Italian population to assess the degree of physical activity participation. The questionnaire classified physical activity as vigorous intensity, moderate intensity and walking, and further obtained information of time and frequency. ${ }^{7}$ IPAQ categories for health, from the IPAQ-scoring protocol (www.ipaq.ki.se): health-enhancing physical activity (HEPA) (reaching recommendations for health-enhancing physical activity) active: vigorous activity at least 3 days per week, totaling at least 1500 MET min per week, or at least 7 days per week of any combination of walking, moderate-intensity or vigorous activities, totaling more than 3000 MET min per week. Minimally active: not HEPA active, but at least 3 days per week of vigorous activity of at least $20 \mathrm{~min}$ per day, or at least 5 days per week 
of moderate-intensity activity or walking at least $30 \mathrm{~min}$ per day, or at least 5 days per week of any combination of walking, moderate-intensity, or vigorous activities, totaling at least 600 MET min per week. Insufficiently active: not belonging to either of the above categories. Total METs (continuous score from the IPAQ-scoring protocol) were calculated as follows: (daily minutes of walking $\times$ days per week with walking $\times 3.3)+($ daily minutes of moderateintensity activity $\times$ days per week with moderate-intensity activity $\times 4.0)+($ daily minutes of vigorous activity $\times$ days per week with vigorous activity $\times 8.0$ ). The MET values were derived from the IPAQ validity and reliability study. ${ }^{8}$

Vigorous activity METs were calculated from the equation daily minutes of vigorous activity $\times$ days per week with vigorous activity $\times 8.0$.

\section{Ankle brachial index measurement}

All patients underwent ABI measurement. The ABI is a simple, noninvasive test, measuring the systolic blood pressure (SBP) from both brachial arteries and from both the dorsalis pedis and posterior tibial arteries after the patient has been at rest in the supine position for $10 \mathrm{~min}$ by using a Doppler device. The ABI of each leg is calculated by dividing the higher of the dorsalis pedis pressure or posterior tibial pressure by the higher of the right or left arm blood pressure.,

\section{Statistical analysis}

SPSS, V.21.0.1 (SPSS Inc, Chicago, Illinois, USA) was used for statistical analysis. Results are presented as mean \pm standard deviation (SD) or frequency expressed as a percentage.

To study the association of physical activity with PAD, we used Cox proportional hazards regression analysis. For these analyses, physical activity was used as continuous and categorical (HEPA activity, minimally active, insufficiently active) variables, in order to obtain the best fitting model. We performed analyses in which we first adjusted for age, BMI, smoking, and adherence to a Mediterranean diet (model 1); and additionally for total cholesterol, HDL cholesterol, SBP, and high sensitivity C-reactive protein (hs-CRP) (model 2).

In interaction analysis, $P$ interaction less than 0.05 was considered to indicate effect modifiers on the association of physical activity with ABI.

\section{Results}

The descriptive results of IPAQ are shown in Table 1 . The MET-minute-per-week distributions were extremely skewed to the right; however, the proportion of women scored as HEPA active was low. The mean Mediterranean diet score was $32.6 \pm 3.3$ (median score was 30.9). The IPAQ Total MET-min per minute was $4970 \pm 4323$ and the IPAQ Vigorous MET-min per minute was $2115 \pm 2196$. The associations between IPAQ groupings and Mediterranean diet were consistent,
Table 1 International Physical Activity Questionnaire categories

\begin{tabular}{lc}
\hline IPAQ categories & Number of patients (percentage of patients) \\
\hline Insufficiently active & $143(33.6 \%)$ \\
Minimally active & $201(47.3 \%)$ \\
HEPA active & $81(19.1 \%)$ \\
\hline
\end{tabular}

HEPA, health-enhancing physical activity; IPAQ, International Physical Activity Questionnaire.

regardless of the way IPAQ was used (HEPA categories, total METs, vigorous): almost all outcome variables showed increasing adherence to a Mediterranean diet from the first (least active) through the fourth group.

Table 2 shows association between clinical characteristics and physical activity (linear regression analysis).

Table 3 illustrates the association of physical activity with ABI value after adjustments for age, BMI, smoking, and adherence to a Mediterranean diet (model 1) and additionally for total cholesterol, HDL cholesterol, SBP and hs-CRP (model 2).

\section{Discussion}

This retrospective analysis was performed to evaluate the relationship between physical activity and asymptomatic PAD in premenopausal women. We observed that our women population was less likely to perform high levels of physical activity. We reported that women who belonged to the highly active group (HEPA active) were also those who had a greater adherence to a Mediterranean diet and had a lower BMI.

In addition, physical activity was inversely associated with preclinical atherosclerosis suggesting a protective effect. The effect of exercise on the prevention of atherosclerosis is not so clear, and because of the multifactorial pathophysiology of plaque, it is plausible that the exercise effects might be modulated by other important factors like diet, antioxidants intake and smoking.

The current recommendation for physical activity in adults and older adults is at least 150 min of moderate-intensity

Table 2 Clinical characteristics of patients and association with physical activity (linear regression analysis)

\begin{tabular}{lccl}
\hline & & $\begin{array}{c}\text { Physical } \\
\text { activity } B \\
\text { Clinical characteristics }\end{array}$ & \\
\hline Mean age (years) & $48.5 \pm 9.2$ & 0.07 & $\mathrm{NS}$ \\
Weight (kg) & $81.2 \pm 4.3$ & 0.20 & 0.05 \\
BMI (mean) & $26.1 \pm 5.6$ & 0.15 & 0.05 \\
Waist circumference (cm) & $87.3 \pm 5.4$ & 0.06 & $\mathrm{NS}$ \\
SBP, mmHg & $124.2 \pm 5.1$ & 0.087 & $\mathrm{NS}$ \\
DBP, mmHg & $88.8 \pm 3.1$ & 0.054 & $\mathrm{NS}$ \\
Smoking (cigarettes/day) & $10 \pm 7$ & 0.16 & 0.05 \\
Alcohol (abstainer) number of patients & 32 & 0.05 & $\mathrm{NS}$ \\
hs-CRP, mg/l (available in 275 patients) & $2.8 \pm 1.3$ & 0.07 & $\mathrm{NS}$ \\
\hline
\end{tabular}

BMI, body mass index; hs-CRP, high sensitivity C-reactive protein; SBP, systolic blood pressure. 
Table 3 Association of physical activity with asymptomatic peripheral arterial disease in adjusted models

\begin{tabular}{lcccc}
\hline ABI & Insufficiently active references & Minimally active HR (95\% Cl) & HEPA active HR (95\% Cl) & Physical activity calculated as total MET HR (95\% Cl) \\
\hline Model 1 & 1 & $0.88(0.37-1.24)$ & $0.82(0.45-0.89)^{*}$ & $0.81(0.32-0.81)^{*}$ \\
Model 2 & 1 & $0.97(0.44-1.29)$ & $0.89(0.24-0.91)^{*}$ & $0.85(0.42-0.87)^{* *}$ \\
\hline
\end{tabular}

$\mathrm{ABI}$, ankle-brachial index; $\mathrm{Cl}$, confidence interval. ${ }^{*} P<0.05{ }^{* *} P<0.01$.

physical activity per week. ${ }^{10}$ This is based on a number of systematic reviews and consensus statement, consistently identifying $150 \mathrm{~min}$ per week as providing considerable health benefits, including reduced all-cause mortality, reduced risk factors for chronic diseases, improved cardiovascular fitness and quality of life. ${ }^{10,11}$ Increasing levels of physical activity was associated with a lower risk of mortality and cardiovascular disease. For women in high-income countries in the global burden of disease study, the estimated population attributable risk for mortality was $14 \%$ for smoking, $8 \%$ for overweight, $5 \%$ for physical inactivity, $4 \%$ for low fruit and vegetable intake and $-3 \%$ for alcohol use. ${ }^{12}$ Mokdad et al. ${ }^{13}$ estimated that the population attributable risks for US mortality in 2000 was $18 \%$ for tobacco, $15 \%$ for poor diet and physical inactivity, and $3.5 \%$ for excess alcohol consumption.

The Mediterranean diet is characterized by a high intake of antioxidants from fruit and vegetables and was associated with a lower incidence of atherosclerosis in several studies. ${ }^{14,15}$

The great majority of articles, however, underline the role of a healthy lifestyle that included both a good diet and high level of physical activity.

Diet and physical activity were evaluated in many different population, that is, in postmenopausal women. ${ }^{16}$ For menopause management, it is recommended that lifestyle changes as a primary modality of care including exercise and a diet rich in calcium, fiber, and low in fat, especially saturated fats ${ }^{1,16}$ Dietary calcium intake and physical activity have a major impact on the bone health in postmenopausal women. Physical activity is defined as any bodily movement produced by skeletal muscle that requires energy expenditure, although, it is known that exercise can be more effective. ${ }^{17,18}$ Physical activity needs to be regular and sustained to confer the largest cardiovascular benefits. However, compared with being inactive over time, all patterns of physical activity change had lower all-cause mortality risk estimates. ${ }^{19}$

The ATTICA study suggested that regular exercise in combination with the Mediterranean dietary pattern may have a beneficial effect on the cardiovascular system through another pathophysiological mechanism, that is, their ability to modulate the oxidation process. Specifically, they found that highly active people who exercise several days per week had higher levels of TAC and that the degree of physical activity was positively correlated with TAC. ${ }^{5}$
In a previous article, we found that fruit and vegetable consumption was inversely associated with preclinical atherosclerosis in women. ${ }^{20} \mathrm{~A}$ diet rich in fruit and vegetables leads to a high intake of carotenoids and vitamins. Because of their antioxidant properties, carotenoid, and vitamins prevent the oxidation of cholesterol and other lipids reducing cardiovascular damage. Physical activity has been shown to greatly improve cardiovascular function, in part through the improved bioavailability of nitric oxide, enhanced endogenous antioxidant defense and a lowering of the expression of ROS-forming enzymes. ${ }^{21,22}$ We suppose that physical activity modulates endothelial response to lipids and a synergy with a healthy diet such as a Mediterranean diet improves this positive effect. Endothelial dysfunction, which is a central mechanism leading to CVD and the redox switch could be modulated by physical activity (that acts directly on endothelium) and dietary factors reducing the risk of GVD onset.

\section{Limitation of the study}

The evaluation of physical activity was obtained using a validated self-administered questionnaire. It is well known that population-level assessments of physical activity overestimate the total amount of physical activity, and perceived intensity of physical activity may vary according to individual fitness or obesity level. ${ }^{23}$ In recent years, several apps have been developed in order to objectively measure the physical activity performed by an individual. Further studies based on accelerometers and phone apps will increase our knowledge. In conclusion, we can suggest that physical activity plays an important role in the prevention of atherosclerosis in premenopausal women.

\section{Acknowledgements Conflicts of interest}

There are no conflicts of interest.

\section{References}

1 WHO 2011 Cardiovascular Diseases (CVDs). World Health Organization. Available at: http://www.who.int/mediacentre/factsheets/fs317/en/ index.html. September 2011; Vol. Fact Sheet Number 317.

2 Mattioli AV, Palmiero P, Manfrini O, et al. Mediterranean diet impact on cardiovascular diseases: a narrative review. J Cardiovasc Med (Hagerstown) 2017; 18:925-935.

3 Maslov PZ, Schulman A, Lavie CJ, et al. Personalized exercise dose prescription. Eur Heart J 2018; 39:2346-2355.

4 Mattioli AV, Coppi F, Migaldi M, et al. Relationship between Mediterranean diet and asymptomatic peripheral arterial disease in a population of premenopausal women. Nutr Metab Cardiovasc Dis 2017; 27:985-990.

5 Kavouras SA, Panagiotakos DB, Pitsavos C, et al. Physical activity and adherence to Mediterranean diet increase total antioxidant capacity: the ATTICA study. Cardiol Res Pract 2010; 2011:248626. 
6 Mattioli AV, Mattioli AV, Miloro C, et al. Adherence to Mediterranean diet and intake of antioxidants influence spontaneous conversion of atrial fibrillation. Nutr Metab Cardiovasc Dis 2013; 23:115-121.

7 Mannocci A, Di Thiene D, Del Cimmuto A, et al. International Physical Activity Questionnaire: validation and assessment in an Italian sample. Ital J Public Health 2010; 7:369-376.

8 Craig CL, Marshall AL, Sjöström M, et al. International Physical Activity Questionnaire: 12-country reliability and validity. Med Sci Sports Exerc 2003; 35:1381-1395.

9 Ciccone MM, Bilianou E, Balbarini A, et al. Task force on: 'Early markers of atherosclerosis: influence of age and sex'. J Cardiovasc Med (Hagerstown) 2013; 14:757-766.

10 Dibben GO, Dalal HM, Taylor RS, et al. Cardiac rehabilitation and physical activity: systematic review and meta-analysis. Heart 2018; 104:1394-1402.

11 Chief Medical Officers. Start active, stay active: a report on physical activity for health from the four home countries. 2011. Available at: https://www. gov. uk/government/uploads/system/uploads/attachment_data/file/ 216370/dh_128210.Pdf.

12 Ezzati M, Vander Hoorn S, Lopez AD, et al. Comparative quantification of mortality and burden of disease attributable to selected risk factors. In: Lopez AD, Mathers CD, Ezzati M, Jamison DT, Murray CJL, editors. Global burden of disease and risk factors. New York: Oxford University Press; 2006. p. 24168.

13 Mokdad AH, Marks JS, Stroup DF, et al. Actual causes of death in the United States. JAMA 2004; 291:1238-1245.

14 Mattioli AV, Migaldi M, Farinetti A. Coffee in hypertensive women with asymptomatic peripheral arterial disease: a potential nutraceutical effect. J Cardiovasc Med (Hagerstown) 2018; 19:183-185.
15 Mattioli AV, Bonatti S, Melotti R, et al. Atrial stunning, inflammation and nutritional status after cardioversion from atrial fibrillation. Int J Cardiol 2008; 129:344-347.

16 Ranasinghe C, Shettigar PG, Garg M. Dietary intake, physical activity, and body mass index among postmenopausal women. J Mid-life Health 2017; 8:163-169.

17 Nelson DB, Sammel MD, Freeman EW, et al. Effect of physical activity on menopausal symptoms among urban women. Med Sci Sports Exerc 2008; 40:50-58.

18 Owens JF, Matthews KA, Räikkönen K, et al. It is never too late: change in physical activity fosters change in cardiovascular risk factors in middleaged women. Prev Cardiol 2003; 6:22-28.

19 Moholdt T, Lavie CJ, Nauman J. Sustained physical activity, not weight loss, associated with improved survival in coronary heart disease. J Am Coll Cardiol 2018; 71:1094-1101.

20 Mattioli AV, Coppi F, Migaldi M, et al. Fruit and vegetables in hypertensive women with asymptomatic peripheral arterial disease. Clin Nutriion ESPEN 2018; 27:110-112.

21 Varadharaj S, Kelly OJ, Khayat RN, et al. Role of dietary antioxidants in the preservation of vascular function and the modulation of health and disease. Front Cardiovasc Med 2017; 4:64.

22 Gliemann L, Nyberg M, Hellsten Y. Nitric oxide and reactive oxygen species in limb vascular function: what is the effect of physical activity? Free Radic Res $2014 ;$ 48:71-78.

23 Tucker JM, Welk GJ, Beyler NK. Physical activity in U.S. adults compliance with the physical activity guidelines for Americans. Am J Prev Med 2011; 40:454-461. 